\title{
Influence d'un coefficient de frottement dépendant de la profondeur sur la propagation des vagues sur un lit poreux
}

\author{
Philippe Sergent ${ }^{1}$, Denis Duhamel ${ }^{2}$ \\ ${ }^{1}$ CETMEF, LHN, Laboratoire commun UTC-CETMEF-CNRS, 2, bd. \\ Gambetta, BP 60039, 60321 Compiègne cedex \\ ${ }^{2}$ ENPC, LAMI, Unité Mixte ENPC-LCPC, Institut Navier, 6/8 av. \\ Blaise Pascal, Cité Descartes, Champs-sur-Marne, 77455 Marne la \\ Vallée cedex 2
}

Résumé. De nombreuses structures maritimes sont formées de digues à talus perméables et submersibles. La plupart des modèles numériques traitant de la propagation des vagues sur un lit poreux linéarise l'équation de Forchheimer sous l'hypothèse de travail équivalent de Lorentz. Le coefficient de frottement $f$ est trouvé par itération et toujours moyenné sur le volume de la structure. Dans cet article, nous écrivons et résolvons la relation de dispersion pour un coefficient de frottement qui est variable sur la profondeur. Nous montrons qu'un coefficient de frottement constant est seulement valable pour une profondeur d'eau $h_{p}$ et une longueur d'onde $L$ avec $h_{p} / L<1 / 10$. Au-dessus de ce seuil, les erreurs sur la partie imaginaire du nombre d'onde ne sont pas négligeables et atteignent $20 \%$ de la valeur du pic. Les calculs avec un coefficient de frottement constant ou dépendant de la profondeur produisent une décroissance différente de la hauteur de houle.

\begin{abstract}
Many maritime structures are made of submerged permeable rubble mound breakwaters. Most numerical models dealing with wave propagation on a porous bed linearize Forchheimer's equation through Lorentz's hypothesis of equivalent work. The friction coefficient $f$ is found by iteration and always averaged on the structure volume. In this paper we write and solve the dispersion relationship for a friction coefficient that is variable on the water depth. It is shown that a constant friction coefficient is only valid for water depth $h_{p}$ and wavelength $L$ with $h_{p} / L<1 / 10$. Above this threshold, the errors on the imaginary part of the wavenumber are not negligible and reach $20 \%$ of the peak value. Calculations with constant and depth-dependent friction coefficient produce a different decay of waveheight.
\end{abstract}

Mots clés. Lit poreux, houle, relation de dispersion, bicouche, éléments finis.

\section{Introduction}

Les structures maritimes de protection des ports contre la houle sont des digues à talus en enrochements, des digues verticales ou des digues composites. Elles arrêtent la houle par dissipation locale de leur énergie ou par réflexion vers le large. Les digues à talus sont formées en particulier d'un empilement d'enrochements qui monte jusqu'à la surface libre. Les structures de protection du 
trait de côte comme les brise-lames détachés ou les épis constituent également des ouvrages en enrochements émergés ou submersibles.

De nombreux modèles ont été développés pour décrire la transformation des vagues sur un fond poreux de profondeur variable. Les premiers modèles théoriques ont été présentés par Sollit et Cross ${ }^{10}$ qui ont évalué la transmission et la réflexion des vagues en présence d'un brise-lames perméable et ont validé les résultats avec des données expérimentales. L'écoulement est modélisé par l'équation de Forchheimer qui est linéarisée à l'aide de l'hypothèse de Lorentz de travail équivalent. Madsen ${ }^{6}$ et Dalrymple et al. ${ }^{2}$ ont mis au point des modèles voisins appliqués à des brises-lames émergés.

Des modèles numériques tridimensionnels ou bidimensionnels vertical prenant en compte des couches poreuses ont aussi été présentés. $\mathrm{Gu}$ et $\mathrm{Wang}^{3}$ résolvent un écoulement potentiel à l'aide de la méthode intégrale d'éléments de frontière et étudient l'interaction des vagues avec un brise-lames composite dont la base est constituée d'enrochements multicouches. Ropert ${ }^{8}$ étudie également l'écoulement à l'intérieur d'un caisson Jarlan posé sur une base en enrochements à l'aide d'un modèle bidimensionnel vertical d'écoulement potentiel aux éléments finis.

Parmi les modèles intégrés sur la verticale, le modèle de Boussinesq de Cruz et al. ${ }^{1}$ étendu à la transformation des vagues sur un lit poreux a été validé sur des données expérimentales de transformation de la houle sur une barre poreuse triangulaire et autour de l'ouverture d'un brise-lames poreux submersible. Les modèles horizontaux les plus communs sont les modèles elliptiques dérivés de l'équation de pentes douces de Berkhoff. Rojanakamthorn et al. ${ }^{7}$ ont les premiers adapté l'équation elliptique pour des brise-lames submersibles poreux en incluant le phénomène de déferlement. Losada et $a l .^{5}$ ont développé aussi l'équation de pente douce pour l'évolution des vagues sur un brise-lames poreux submersible et ont mené une analyse complète des résultats intégrant les effets d'oblicité, de géométrie du brise-lames et des caractéristiques du matériau poreux. Silva et al. ${ }^{9}$ ont ajouté de nouveaux termes à l'équation de pente douce étendue au milieu poreux de façon à prendre en compte des profondeurs rapidement variables.

Dans tous ces travaux, le coefficient de frottement $f$ est obtenu numériquement par itérations et est choisi moyenné sur le volume. Houari et $a l^{4}{ }^{4}$ utilisent un coefficient de frottement variable sur un plan horizontal mais moyenné sur l'axe vertical. L'objectif de ce papier est d'évaluer les variations de ce coefficient de frottement sur l'axe vertical. Une nouvelle procédure est proposée pour évaluer l'influence de ces variations sur le nombre d'onde et sur l'atténuation de la hauteur de houle se propageant sur un lit poreux. Nous écrivons d'abord le modèle de pression dans un milieu poreux. Nous présentons ensuite le système d'équations pour un bicouche et une nouvelle méthode pour résoudre la relation de dispersion pour un coefficient de frottement hétérogène. L'influence de la discrétisation est étudiée avec deux cas de validation: un milieu fluide et un bicouche avec un coefficient de frottement constant. Nous présentons enfin le cas d'application d'un bicouche avec un coefficient de frottement variable en étudiant l'atténuation de la hauteur de houle se propageant sur le fond poreux. 


\section{Milieu poreux}

Le milieu poreux saturé est composé de deux phases : un squelette rigide et un fluide remplissant l'espace interstitiel. Le milieu poreux est inerte : il $\mathrm{n}$ ' $\mathrm{y}$ a pas de création de matière pour les deux phases. Le squelette est supposé immobile et le fluide incompressible. Les variables dépendent de la position mais leur dépendance est omise pour simplifier les équations. Les équations de conservation de la masse et de la quantité de mouvement sont respectivement :

$$
\operatorname{div}\left(\varepsilon \overrightarrow{V_{s}}\right)=0
$$

avec $\varepsilon$ la porosité, $\overrightarrow{V_{s}}$ la vitesse interstitielle, $\varepsilon \overrightarrow{V_{s}}$ la vitesse de filtration.

$$
\frac{\partial \overrightarrow{V_{s}}}{\partial t}=-\frac{1}{\rho} \vec{\nabla}(p+\rho g z)-\overrightarrow{F_{r}}-\vec{F}_{i}
$$

où $\rho$ représente la densité du fluide, $p$ la pression, $z$ la coordonnée verticale mesurée positivement vers le haut et $\vec{\nabla}$ l'opérateur Gradient.

Le terme de résistance $\overrightarrow{F_{r}}$ s'exprime en fonction de la vitesse interstitielle :

$$
\overrightarrow{F_{r}}=\frac{v \varepsilon}{K} \overrightarrow{V_{S}}+\frac{C_{f} \varepsilon^{2}}{\sqrt{K}}\left|\overrightarrow{V_{S}}\right| \overrightarrow{V_{S}}
$$

où $v$ est la viscosité, $C_{f}$ le coefficient de résistance turbulente et $K$ la perméabilité du milieu poreux.

Le terme de résistance inertielle $\vec{F}_{i}$ dépend du coefficient de masse virtuelle $C_{m}$ et de l'accélération dans le sens de l'écoulement (Sollit et $\operatorname{Cross}^{10}$ ).

$$
\overrightarrow{F_{i}}=\frac{1-\varepsilon}{\varepsilon} C_{m} \frac{\partial \overrightarrow{V_{S}}}{\partial t}
$$

Le coefficient $C_{m}$ est estimé pour un grain individuel de forme régulière mais reste en général inconnu pour un ensemble de grains composant un corps poreux. Remplaçant les expressions $\overrightarrow{F_{r}}$ et $\overrightarrow{F_{i}}$ dans l'équation de conservation de la quantité de mouvement (2), nous obtenons l'équation de Forchheimer qui est utilisée en général par les ingénieurs pour décrire l'écoulement dans un milieu poreux soumis à l'action des vagues.

$$
\mathrm{S} \frac{\partial \overrightarrow{V_{s}}}{\partial t}=-\frac{1}{\rho} \vec{\nabla}(p+\rho g z)-\frac{v \varepsilon}{K} \vec{V}_{s}-\frac{C_{f} \varepsilon^{2}}{\sqrt{K}}\left|\overrightarrow{V_{s}}\right| \overrightarrow{V_{s}}
$$

avec $S=1+\frac{1-\varepsilon}{\varepsilon} C_{m}$. Le terme linéaire, le terme de Darcy, gouverne l'équation pour les petits nombres de Reynolds dans un milieu poreux fin. Le terme nonlinéaire, le terme de Forchheimer, est une extension du terme de Darcy pour des milieux poreux plus grossiers avec de grands nombres de Reynolds pour lesquels l'effet d'inertie dépasse l'effet de viscosité.

\subsection{Linéarisation}


En considérant une excitation monochromatique, les termes dissipatifs, linéaires ou non-linéaires de l'équation de Forchheimer sont remplacés par un terme équivalent linéaire :

$$
\frac{v \varepsilon}{K} \vec{V}_{s}+\frac{C_{f} \varepsilon^{2}}{\sqrt{K}}\left|\overrightarrow{V_{s}}\right| \overrightarrow{V_{s}} \rightarrow f \omega \overrightarrow{V_{s}}
$$

Dans cette expression $f$ représente un coefficient de frottement ou d'atténuation, $\omega$ est la pulsation de l'excitation monochromatique de période $T$. L'évaluation du coefficient $f$ repose sur l'hypothèse de Lorentz de travail équivalent qui consiste à égaler, sur une période de houle, le travail moyen du terme non-linéaire avec le travail moyen d'un terme linéaire. Le terme $f$ est :

$$
f \omega=\frac{v \varepsilon}{K}\left(1+\frac{C_{f} \varepsilon \sqrt{K}}{v} \bar{f}\right)
$$

avec $\quad \bar{f}=\frac{\int_{0}^{T}\left|\overrightarrow{V_{s}}\right|^{3} d t}{\int_{0}^{T}\left|\overrightarrow{V_{s}}\right|^{2} d t}=\frac{8}{3 \pi} \sqrt{a+b}\left[E(m)-\frac{1}{4}\left(1-\frac{b}{a}\right) K(m)\right]$ et $\quad a=\frac{1}{T} \int_{0}^{T}\left|\overrightarrow{V_{s}}\right|^{2} d t$, $b=\max _{t \in[0, T}\left(\left|\vec{V}_{s}\right|^{2}-a\right), m=\frac{2 b}{a+b} . K(m)$ et $E(m)$ représentent respectivement les intégrales elliptiques de première et seconde espèce.

\subsection{Equations pour une excitation monochromatique}

Les solutions monochromatiques s'écrivent avec $p^{*}=p+\rho g z$ :

$$
\begin{aligned}
\overrightarrow{V_{s}} & =\operatorname{Re}\left(\overrightarrow{\mathrm{V}} \mathrm{e}^{-\mathrm{j} \omega \mathrm{t}}\right) \\
p^{*} & =\operatorname{Re}\left(\mathrm{Pe}^{-\mathrm{j} \omega \mathrm{t}}\right)
\end{aligned}
$$

L'expression linéaire de la conservation de la quantité de mouvement en fonction des variables $(\vec{V}, \mathrm{P})$ et du coefficient de frottement $f$ s'écrit :

$$
-j \omega S \vec{V}=-\frac{1}{\rho} \overrightarrow{\nabla P}-f \omega \vec{V}
$$

L'équation peut aussi s'écrire sous la forme donnant la vitesse de filtration :

$$
\varepsilon \vec{V}=-\frac{1}{\rho j \omega} \beta \overrightarrow{\nabla P}
$$

avec $\beta=\frac{\varepsilon}{S+j f}$. L'équation de conservation de la masse permet d'obtenir l'équation finale :

$$
\operatorname{div}(\beta \vec{\nabla} P)=0
$$

Cette équation, avec les conditions aux limites à la surface libre et sur le fond rigide, définit un modèle avec la pression comme inconnue principale. Ce modèle représente la propagation de vagues linéaires monochromatiques au-dessus d'un fond poreux. La résolution de cette équation par un schéma itératif donne les 
champs de vitesse et de pression dans le milieu poreux. Cette équation est commune aux milieux fluide et poreux en considérant un milieu hétérogène avec une fonction $\beta$ variable spatialement. Cette fonction est constante et égale à 1 dans le milieu fluide et est variable dans le milieu poreux. De façon à simplifier les équations, nous choisissons un écoulement suivant l'axe $x$. Pour une excitation monochromatique nous écrivons des nouvelles expressions condensées pour les coefficients $a$ et $b$ :

$$
\begin{aligned}
& \mathrm{a}=\frac{1}{2}\left(\left|V_{x}\right|^{2}+\left|V_{z}\right|^{2}\right) \\
& b=\frac{1}{2}\left|V_{x}^{2}+V_{z}^{2}\right|
\end{aligned}
$$

avec $V_{x}$ et $V_{z}$ les composantes horizontale et verticale respectivement de la vitesse complexe $\vec{V}$.

\section{Théorie du bicouche}

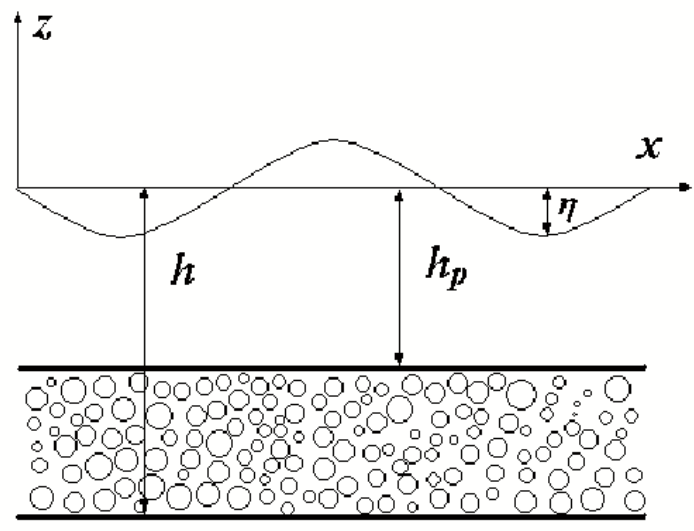

Figure 1 : Schéma d'un bicouche.

Un bicouche, représenté sur la figure 1, est composé d'un milieu fluide submergeant un milieu poreux qui repose sur un fond rigide. Les axes orthogonaux $O x$ et $\mathrm{O} y$ définissent un plan horizontal qui coïncide avec la surface libre. La profondeur constante du fluide est notée $h_{p}$ et la profondeur constante totale est notée $h$.

\subsection{Système d'équations}

Le déplacement de la surface libre $\eta(x, t)$ est écrit pour des solutions monochromatiques :

$$
\eta=\operatorname{Re}\left(\mathrm{Ae}^{-\mathrm{j} \omega \mathrm{t}}\right)
$$

A la surface libre, les conditions linéaires dynamiques et cinématiques avec l'équation de Bernoulli linéarisée donnent en $z=0$ :

$$
\frac{\partial P}{\partial z}-\frac{\omega^{2}}{g} P=0 \quad \text { et } \quad P=\rho g A
$$


Sur le fond plat rigide, la composante verticale de la vitesse $\vec{V}$ est nulle de telle sorte que $\frac{\partial P}{\partial z}=0$.

Le système d'équations satisfait par la pression $P$ est finalement :

$$
\left\{\begin{array}{cc}
\operatorname{div}(\beta \overrightarrow{\nabla P})=0 & -h \leq z \leq 0 \\
\frac{\partial P}{\partial z}-\frac{\omega^{2}}{g} P=0 & z=0 \\
\frac{\partial P}{\partial z}=0 & z=-h
\end{array}\right.
$$

Avec un bicouche, la quantité $\beta$ varie avec le coefficient de frottement $f$. Le coefficient de frottement $f$ est fonction des coefficients $a$ et $b$ qui sont eux-mêmes des fonctions de la vitesse interstitielle $\vec{V}$ et de la pression $P$. Comme la pression $P$ varie spatialement, c'est aussi le cas de $\beta$ qui varie en particulier le long de l'axe vertical.

\subsection{Relation de dispersion}

Nous cherchons une solution du système d'équations (15) sous la forme de variables séparées :

$$
P(x, z)=\phi(x) Z(z)
$$

Dans la couche poreuse et la couche fluide, la première équation du système (15) donne, avec le nombre d'onde $k$ identique dans les deux couches :

$$
\frac{1}{\phi} \frac{\mathrm{d}^{2} \phi}{\mathrm{d} x^{2}}=\frac{\left(\beta Z^{\prime}\right)^{\prime}}{\beta Z}=k^{2}
$$

Le coefficient $\beta$ est moyenné dans chaque couche horizontale de telle sorte qu'il est supposé dépendre seulement de la coordonnée verticale $z$. Nous réécrivons l'équation (17) sous la forme suivante :

$$
\begin{aligned}
& \frac{\mathrm{d}^{2} \phi}{\mathrm{d} x^{2}}+k^{2} \phi=0 \\
& \left(\beta Z^{\prime}\right)^{\prime}-k^{2} \beta Z=0
\end{aligned}
$$

La seconde équation demande la continuité de $\beta Z$ ' et de $Z$.

\subsection{Houle plane incidente d'amplitude $A_{0}$}

Nous choisissons $Z(0)$ égal à 1 ce qui donne $\phi(x)=\rho g A(x)$ avec l'équation (16). L'amplitude de houle vérifie donc également l'équation :

$$
\frac{\mathrm{d}^{2} A}{\mathrm{~d} x^{2}}+k^{2} A=0
$$

Nous choisissons une houle plane incidente se propageant selon l'axe des $\mathrm{x}$ avec une amplitude $A_{0}$ en $x=0$.

$$
A(x)=A_{0} e^{j k x}
$$

\subsection{Relations entre les coefficients $a, b$ et $A_{0}$}


La pression $P$ prend donc la forme finale :

$$
P(x, y, z)=\rho g A_{0} e^{j k x} Z(z)
$$

Avec la célérité c égale à $\frac{\omega}{k}$, nous en déduisons que :

$$
\begin{gathered}
V_{x}=-\frac{\mathrm{g}}{\mathrm{c}} \frac{\beta}{\varepsilon} A_{0} Z(z) e^{j k x} \\
V_{z}=-\frac{\mathrm{g}}{\mathrm{j} \omega} \frac{\beta}{\varepsilon} A_{0} Z(z) e^{j k x} \\
a=\frac{1}{2}\left(\frac{\mathrm{g}}{\omega} \frac{\beta}{\varepsilon}\right)^{2} A_{0}^{2}\left(|k Z(z)|^{2}+\left|Z^{\prime}(z)\right|^{2}\right)\left|e^{j k x}\right|^{2} \\
b=\frac{1}{2}\left(\frac{\mathrm{g}}{\omega} \frac{\beta}{\varepsilon}\right)^{2} A_{0}^{2}\left|k^{2} Z^{2}(z)-Z^{\prime 2}(z)\right|\left|e^{j k x}\right|^{2}
\end{gathered}
$$

Le coefficient $\beta$ est moyenné dans chaque couche horizontale sur un segment de longueur $\left[0, L_{x}\right]$ avec la longueur $L_{x}$ supposée petite de telle sorte que $\left|e^{j k L_{x}}\right| \approx 1$.

\subsection{Procédure de résolution}

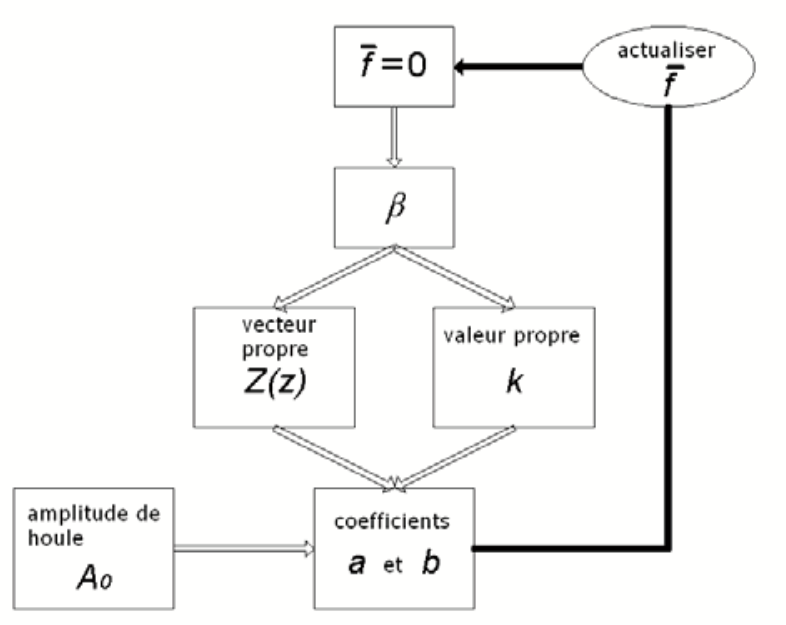

Figure 2 : Procédure de résolution.

La solution de Darcy $(\bar{f}=0)$ est d'abord calculée. La procédure consiste à trouver le vecteur propre $Z(z)$ associé avec la première valeur propre et le nombre d'onde complexe $k$. En donnant l'amplitude de houle incidente $A_{0}$, les coefficients $\mathrm{a}$ et $\mathrm{b}$ sont calculés et utilisés pour actualiser la valeur $\operatorname{de} \bar{f}$. Cette opération est répétée aussi longtemps que la différence relative de $\bar{f}$ entre deux itérations successives excède $10^{-6}$. Le schéma de la procédure est synthétisé sur la figure 2 .

\subsection{Valeurs propres et vecteurs propres}

Le système (15) est réécrit en fonction de la fonction $Z$ : 


$$
\left\{\begin{array}{cc}
\left(\beta Z^{\prime}\right)^{\prime}-k^{2} \beta Z=0 & -h \leq z \leq 0 \\
Z^{\prime}-\frac{\omega^{2}}{g} Z=0 & z=0 \\
Z^{\prime}=0 & z=-h
\end{array}\right.
$$

La pulsation $\omega$ et la quantité $\beta$, fonction de $z$, sont données. Nous cherchons les vecteurs propres $Z$ et valeurs propres $\mathrm{k}$ du système (24) appelé aussi problème de Sturm-Liouville. Dans ce système, les fonctions $Z$ ' et $\beta$ ne sont pas continues. Il y a en particulier un saut de $Z$ ' et de $\beta$ à l'interface entre le milieu fluide et le milieu poreux. Cependant $\beta Z$ ' et $Z$ sont des fonctions continues. Cela implique que $\varepsilon V_{z}$ et $P$ sont des fonctions continues. La solution du système (26) dans une couche $i$ avec $\beta$ constant par couche est :

$$
Z(z)=A_{i} \cosh (k z)+B_{i} \sinh (k z)
$$

Pour un grand nombre de couches, cette méthode devient complexe et lourde en temps de calcul. C'est la raison pour laquelle nous préférons écrire la forme variationnelle de (26) avec la fonction test $\delta Z$ :

$$
\int_{-h}^{0}\left(\beta Z^{\prime}\right)^{\prime} \delta Z d z-k^{2} \int_{-h}^{0} \beta Z \delta Z=0
$$

Une intégration par parties avec application des conditions aux limites donne :

$$
-\int_{-h}^{0} \beta Z^{\prime} \delta Z^{\prime} d z-k^{2} \int_{-h}^{0} \beta Z \delta Z+\frac{\omega^{2}}{g} Z(0) \delta Z(0)=0
$$

La méthode des éléments finis découpe le domaine $[-h, 0]$ en un nombre fini $N$ de sous-domaines $\Omega_{i}$ avec $\Omega_{i}=\left[(i-1) \frac{h}{N}, i \frac{h}{N}\right]$. Nous choisissons des éléments linéaires L2 avec deux nœuds et deux degrés de liberté. Les fonctions solutions $Z$ et fonctions test $\delta Z$ sont représentées par leur valeur nodale $Z_{n}$ et $\delta Z_{n}(n=1,2)$ avec

$$
Z=\left\langle N_{n}(\xi)\right\rangle\left\{Z_{n}\right\} \quad \delta Z=\left\langle N_{n}(\xi)\right\rangle\left\{\delta Z_{n}\right\}
$$

où $\xi$ est la variable réduite associée au domaine de référence $A=[0,1]$. La notation $<>$ représente la transposée du vecteur \{\} . Les fonctions de forme $N_{1}$ et $N_{2}$ sont égales à $1-\xi$ et $\xi$ respectivement. La forme variationnelle (29) prend la forme suivante :

$$
\begin{gathered}
-\frac{N}{h} \sum_{i=1}^{N} \beta_{i}\left\langle\delta Z_{n}\right\rangle\left[\int_{A}\left\{N_{n}^{\prime}(\xi)\right\}\left\langle N_{n}^{\prime}(\xi)\right\rangle d \xi\right]\left\{Z_{n}\right\} \\
-k^{2} \frac{h}{N} \sum_{i=1}^{N} \beta_{i}\left\langle\delta Z_{n}\right\rangle\left[\int_{A}\left\{N_{n}(\xi)\right\}\left\langle N_{n}(\xi)\right\rangle d \xi\right]+\frac{\omega^{2}}{g} \delta Z^{1} Z^{1}=0
\end{gathered}
$$

où $\beta$ prend une valeur constante $\beta_{i}$ sur chaque élément. Les valeurs nodales des fonctions sont aussi notées $\left.Z^{i}=Z\left(-(i-1) \frac{h}{N}\right)\right)$ et $\left.\delta Z^{i}=\delta Z\left(-(i-1) \frac{h}{N}\right)\right)$ $(i=1, \ldots, N+1)$. Nous réécrivons l'équation (31) sous la forme suivante :

$$
\sum_{i j} \delta Z^{j}\left(-\frac{N}{h} K_{i j}-k^{2} \frac{h}{N} M_{i j}+R_{i j}\right) Z^{i}=0
$$


avec

$$
\mathrm{K}=\left[\begin{array}{ccccccccc}
\beta_{1} & -\beta_{1} & . . & 0 & 0 & 0 & . . & 0 & 0 \\
-\beta_{1} & \beta_{1}+\beta_{2} & . . & 0 & 0 & 0 & . . & 0 & 0 \\
: & : & : & : & : & : & : & : & : \\
0 & 0 & . . & \beta_{j-1}+\beta_{j} & -\beta_{j} & 0 & . . & 0 & 0 \\
0 & 0 & . . & -\beta_{j} & \beta_{j}+\beta_{j+1} & -\beta_{j+1} & . . & 0 & 0 \\
0 & 0 & . . & 0 & -\beta_{j+1} & \beta_{j+1}+\beta_{j+2} & . . & 0 & 0 \\
: & : & : & : & : & : & : & : & : \\
0 & 0 & . . & 0 & 0 & 0 & . . & \beta_{N-1}+\beta_{N} & -\beta_{N} \\
0 & 0 & . . & 0 & 0 & 0 & . . & -\beta_{N} & \beta_{N}
\end{array}\right]
$$

$$
\mathrm{M}=\frac{1}{3}\left[\begin{array}{ccccccccc}
\beta_{1} & \frac{\beta_{1}}{2} & . . & 0 & 0 & 0 & . . & 0 & 0 \\
\frac{\beta_{1}}{2} & \beta_{1}+\beta_{2} & . . & 0 & 0 & 0 & . . & 0 & 0 \\
: & : & : & : & : & : & : & : & : \\
0 & 0 & . . & \beta_{j-1}+\beta_{j} & \frac{\beta_{j}}{2} & 0 & . . & 0 & 0 \\
0 & 0 & . . & \frac{\beta_{j}}{2} & \beta_{j}+\beta_{j+1} & \frac{\beta_{j+1}}{2} & . . & 0 & 0 \\
0 & 0 & . . & 0 & \frac{\beta_{j+1}}{2} & \beta_{j+1}+\beta_{j+2} & . . & 0 & 0 \\
: & : & : & : & : & : & : & : & : \\
0 & 0 & . . & 0 & 0 & 0 & . . & \beta_{N-1}+\beta_{N} & \frac{\beta_{N}}{2} \\
0 & 0 & . . & 0 & 0 & 0 & . . & \frac{\beta_{N}}{2} & \beta_{N}
\end{array}\right]
$$

et $R_{i j}=\frac{\omega^{2}}{g} \delta_{i 1} \delta_{j 1}$ où $\delta_{i j}$ est le symbole de Kronecker.

Notant $\delta \mathrm{Z}$ et $\mathrm{Z}$ les composantes de vecteurs $\delta Z_{i}$ et $Z_{i}$ respectivement. Comme l'équation (32) est valable pour n'importe qu'elle fonction test $\delta Z$, cela conduit à la relation suivante :

$$
\left[\mathbf{K}+\left(\frac{k h}{N}\right)^{2} \mathbf{M}-\frac{h}{N} \mathbf{R}\right] \mathbf{Z}=\mathbf{0}
$$

Ce système représente un problème de valeur propre généralisé. Il est résolu par des routines standard développées pour ce type de problème et qui donnent une série de valeurs propres $k$ et de vecteurs propres $\mathbf{Z}$. Dans le reste de l'article nous présentons seulement les résultats concernant le mode propagatif mais la méthode peut aussi bien trouver les racines des modes évanescents. 


\section{Validation de la relation de dispersion}

\subsection{Cas particulier $\mathrm{A}$ : simple milieu fluide}

Le cas particulier d'un simple milieu fluide $(\beta=1)$ est choisi de façon à étudier l'influence de la discrétisation sur la précision de la relation de dispersion bien connue $\omega^{2}=g k \tanh (k h)$.

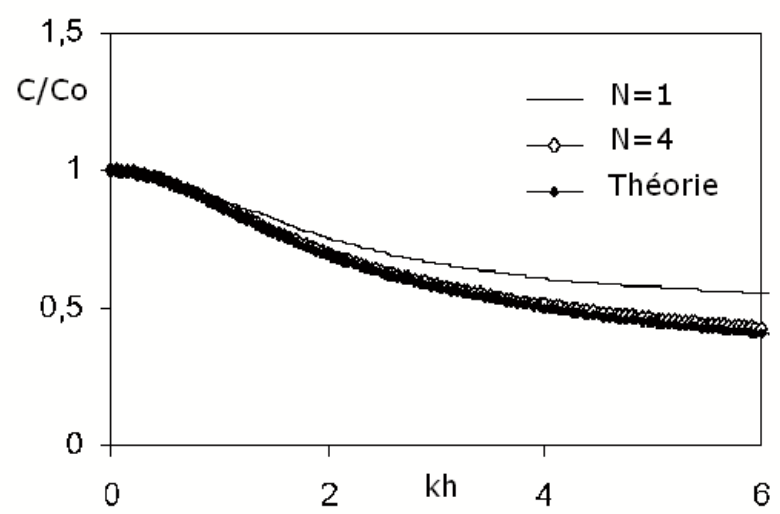

Figure 3 : Cas A : relation de dispersion.

La figure 3 présente la relation de dispersion théorique et les approximations avec un et quatre éléments respectivement. Quatre éléments sont suffisants pour bien approximer la relation de dispersion pour $k h \leq 6$.

\subsection{Cas particulier $B$ : bicouche avec valeur de $\beta$ constante}

Un second cas particulier est le bicouche avec une valeur de $\beta$ constante. Pour le cas B, la valeur de $\beta$ est calculée avec $f=1, S=1, \varepsilon=0,4, h=1$ et $h_{p}=0,25$. La relation de dispersion est présentée par Rojanakamthorn et al. ${ }^{7}$.

$$
\omega^{2}=g k \tanh \left(k h_{p}\right)+\beta \tanh \left(k\left(h-h_{p}\right)\right)\left(g k-\omega^{2} \tanh \left(k h_{p}\right)\right)
$$
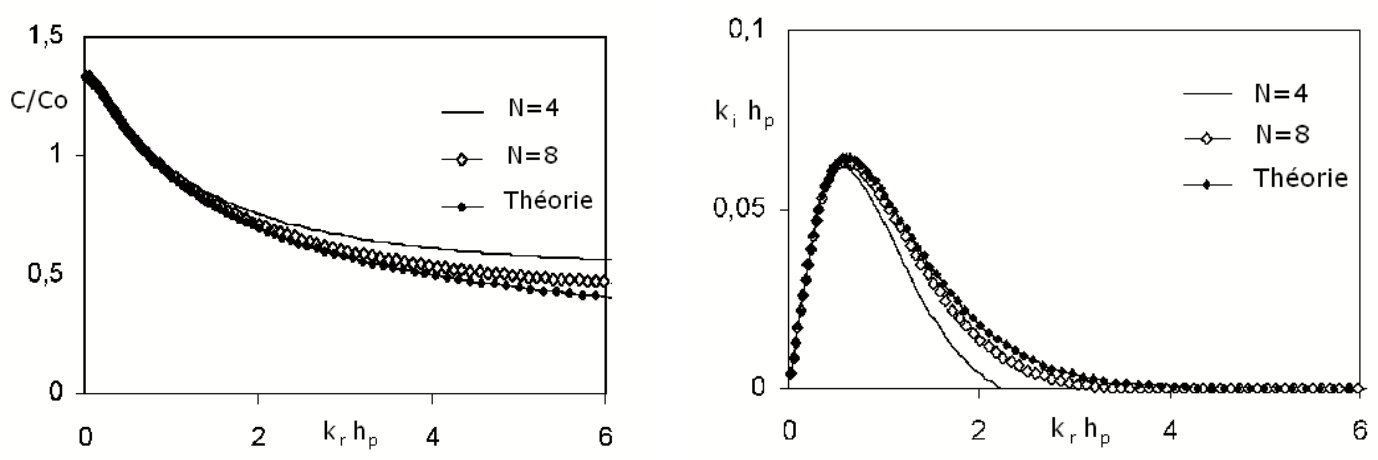

Figure 4 : Cas B : relation de dispersion (gauche) et dissipation (droite).

La relation de dispersion et la partie imaginaire du nombre d'onde sont présentées sur la figure 4. Ce cas B demande plus d'éléments que le premier cas car les variations verticales de la pression sont plus importantes. Même avec huit 
éléments, la relation de dispersion numérique et les courbes de dissipation diffèrent des relations théoriques. Pour des houles courtes (ici $k h \geq 4$ ), les cas A et $\mathrm{B}$ deviennent équivalents car le fond poreux n'affecte plus la propagation des vagues et aucune dissipation ne se produit.

\section{Application}
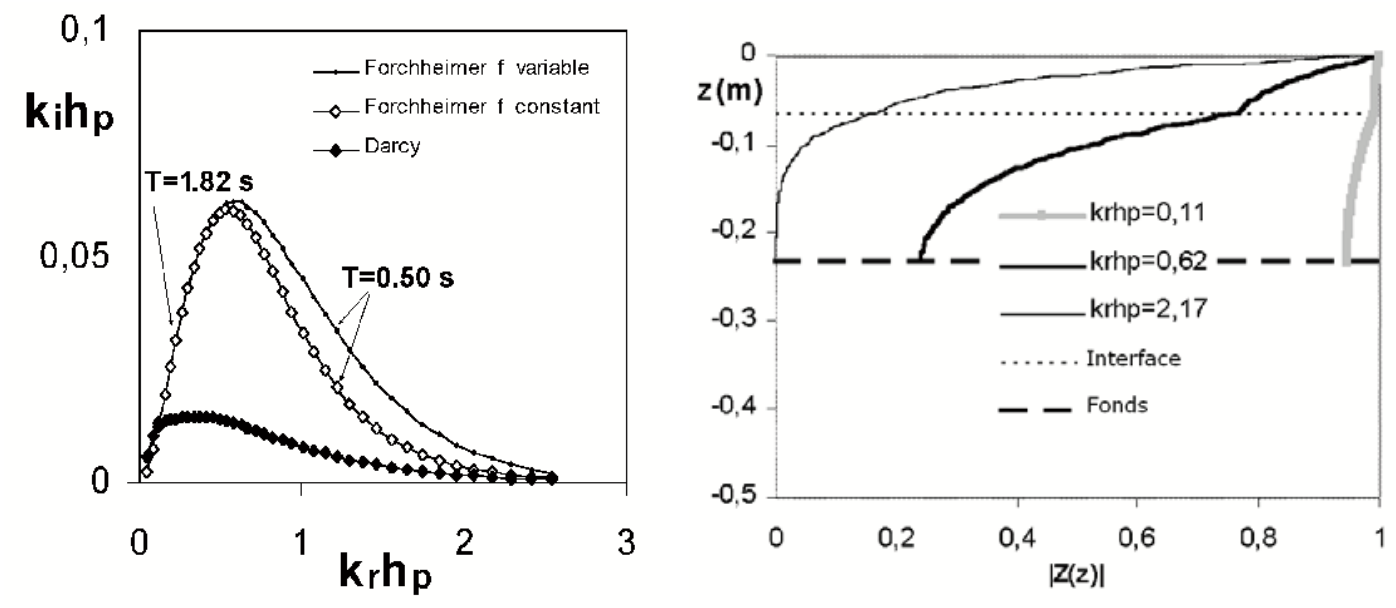

Figure 5 : Cas $\mathrm{C}$ : dissipation (gauche) et variations de $Z$ (droite).

Prenant le cas expérimental (a) de Rojanakamthorn et al. ${ }^{7}$ avec les valeurs suivantes : $A_{0}=2,2 \mathrm{~cm} ; K=3,7710^{-7} \mathrm{~m} ; T=1,82 \mathrm{~s} ; C_{f}=0,332 ; \varepsilon=0,39$; $N=72 ; h_{p}=6,5 \mathrm{~cm} ; h=23,4 \mathrm{~cm}$, nous avons déterminé les variations $\mathrm{du}$ coefficient de frottement $f$. Nous trouvons presque le même coefficient de frottement moyenné que les auteurs. Les variations de $f$ sur la verticale représentent quant à elles $10 \%$ de la valeur moyenne. Le coefficient de frottement est plus grand à l'interface poreux-fluide que sur le fond rigide.

Nous avons testé trois approximations: l'approximation de Darcy, l'approximation de Forchheimer avec un coefficient de frottement constant et l'approximation de Forchheimer avec un coefficient de frottement variable. En ce qui concerne la relation de dispersion, aucune différence significative n'a été relevée entre les deux approximations de Forchheimer. Sur la figure 5 est présentée la partie imaginaire du nombre d'onde avec les trois mêmes approximations. Cette valeur est reliée à la dissipation des vagues se propageant sur un lit poreux. Des différences significatives sont relevées entre les deux approximations de Forchheimer lorsque $h_{p} / L$ est supérieur à $1 / 10$ avec $L$ la longueur d'onde. Ces différences peuvent atteindre $20 \%$ de la valeur de pic. $\mathrm{Au}$ contraire, les approximations de Darcy et de Forchheimer donnent des résultats similaires lorsque $h_{p} / L$ est supérieur à $1 / 3$ car la pénétration des vagues dans le milieu poreux est faible pour ces longueurs d'onde. En résumé, les variations verticales du coefficient de frottement dans les équations de Forchheimer ont un effet pour des profondeurs intermédiaires lorsque $1 / 10<h_{p} / L<1 / 3$.

Sur la figure 5 sont également présentées les variations verticales de la fonction $Z$. Pour des ondes longues, cette fonction est presque constante et égale à 1 quelle 
que soit la hauteur de houle. Cela explique pourquoi l'hypothèse d'un coefficient de frottement constant est valable. Pour des ondes courtes, cette fonction varie rapidement dans le milieu fluide de telle sorte que la fonction $Z$ est proche de zéro dans le milieu poreux et cela explique aussi pourquoi peu de différences sont relevées entre les lois de Darcy et Forchheimer. Pour un nombre d'onde intermédiaire (ici $k_{r} h_{p}=0,62$ ), la fonction $Z$ n'est plus constante dans le milieu poreux et cela met en évidence les limites de l'hypothèse d'un coefficient de frottement constant. Comme déjà mentionné plus haut, le saut du coefficient $\beta$ à l'interface entre le milieu fluide et le milieu poreux entraîne un saut de $Z$ ' car la composante verticale de la vitesse de filtration est continue.

\section{Conclusions}

Etudiant la propagation des vagues sur un milieu poreux, l'équation de Forchheimer est linéarisée à l'aide de l'hypothèse de Lorentz du travail équivalent. Utilisant une forme variationnelle, nous écrivons et résolvons la relation de dispersion pour un coefficient de frottement $f$ qui est variable suivant la verticale. Avec deux cas-tests, nous trouvons que dix éléments sur la profondeur d'eau fournissent une bonne approximation de la relation de dispersion. Nous montrons ensuite que les variations verticales du coefficient de frottement dans les équations de Forchheimer ont un effet sur la dissipation de la houle pour des profondeurs intermédiaires pour lesquelles $1 / 10<h_{p} / L<1 / 3$.

\section{$\underline{\text { Références }}$}

1 Cruz E.C., Isobe M., Watanabe A. (1997). Boussinesq equations for wave transformation on porous beds. Coast. Eng., 30, 125-156.

2 Dalrymple R.A., Losada M.A., Martin P.A. (1991). Reflection and transmission from porous structures under oblique wave attack. J. Fluid Mech., 224, 625-644.

3 Gu G.Z., Wang H. (1992). Numerical modelling for wave energy dissipation within porous submerged breakwaters of irregular sections. Coast. Eng. Conf. Proc., 1189-1199.

4 Houari M., Sergent P., Duhamel D. (2002). Réflexion de la houle sur les ouvrages: modèle de houle à dissipation. $7^{\text {èmes }}$ J. Gén. Civ. Gén. Côtier, 93-103.

5 Losada I.J., Silva R., Losada M.A. (1996). 3D non-breaking regular wave interaction with submerged breakwaters. Coast. Eng. 28, 229-248.

6 Madsen O.S. (1974). Wave transmission through porous structures. J. Waterw., Port, Coast., Ocean Eng. 100 (3), 169-188.

7 Rojanakamthorn S., Isobe M., Watanabe A. (1990). Modelling of wave transformation on submerged breakwater. Coast. Eng. Conf. Proc., 1060-1073.

8 Ropert F. (1999). Modélisation du comportement hydraulique des caissons Jarlan. Thèse de doctorat de l'Université de technologie de Compiègne.

9 Silva R., Salles P., Palacio A. (2002). Linear waves propagating over a rapidly varying finite porous bed. Coast. Eng. 44, pp 239-260.

10 Sollit C.K., Cross R.H. (1972). Wave transmission through permeable breakwater. Coast. Eng. 13, 1827-1846. 